\title{
Bistable vibration energy harvester and SECE circuit: exploring their mutual influence
}

\author{
Thomas Huguet ${ }^{a, b}$, Mickaël Lallart ${ }^{a,}$ and Adrien Badel ${ }^{b}$ \\ ${ }^{a}$ Univ. Lyon, INSA-Lyon, LGEF, EA682, F-69621, Villeurbanne, France \\ ${ }^{\mathrm{b}}$ Univ. Savoie Mont-Blanc, SYMME, F-74000, Annecy, France \\ * corresponding author: mickael.lallart@insa-lyon.fr
}

\begin{abstract}
The present work focuses on vibration energy harvesting to replace chemical batteries as power supplies in wireless autonomous sensors. More particularly, this article deals with nonlinear inertial oscillators and more precisely bistable harvesters, which offer a wide bandwidth compared to linear structures. These harvesters require AC-DC electronic interface to be able to supply wireless sensors or small intermediate storage elements (used to smooth the power demand). However, few studies have investigated the influence of AC-DC circuits on bistable harvesters. This work therefore focuses on developing the first analytical model able to predict the frequency response of a bistable harvester coupled to one of these interfaces: the SECE circuit (Synchronized Electric Charge Extraction). This mathematical model includes subharmonic behaviors, stability analyses to small disturbances and the analyses of the stability robustness of high orbits characterizing their capability to handle external disturbances in real conditions without falling on a low orbit. The model is then validated experimentally with a buckled beam bistable harvester with piezoelectric conversion. The latter shows that the use of SECE circuit leads to multiply the mean extracted power from 1.3 to 2.2 compared to a direct connection to the load. The reachable frequency range is nevertheless divided by 1.5.
\end{abstract}

Keywords: Vibration - Energy harvesting - Bistable - SECE - Analytical model

\section{Introduction}

With the spreading of IoT devices such as wireless sensors and sensor networks, many recent works were devoted to the search for an alternative to the use of chemical batteries for the power supply of autonomous wireless sensors. More particularly, this study focuses on devices able to harvest the ambient vibration energy through electromechanical conversion. Vibration energy is indeed present in many different industrial environments (including indoor environments) where the market of wireless sensors expands for data acquisition, health monitoring, etc.. The use of these vibration energy harvesters instead of chemical batteries will lead to more compact wireless sensors (no more need to embed big quantities of energy with the sensor), with a greater autonomy (no more need to periodically replace the empty batteries during the sensor lifetime), and able to work in more severe environments (high temperature for example). More specifically, it can be noted that energy harvesting can also be used to address the issue of selfdischarge of primary batteries that limits the device's lifespan.

In this context the scientific community has proposed different kind of architectures for these harvesters [1]. In order to conceive devices with a unique point of fixation on the vibration source, studies focused on inertial oscil- lating harvesters. Among them, linear harvesters offer good performances at their resonant frequency. Nevertheless, the output power of these devices rapidly collapses if the frequency of the source slightly changes over time. To deal with this problem, the scientific community proposed to use nonlinear harvesters which possess a wider useful frequency range [2]. This work focuses on a promising and simple solution in terms of bandwidth: the bistable harvester [3].

The interface circuit between the vibration energy harvester and the device that needs to be power supplied is also a key point: the first one produces $\mathrm{AC}$ voltage as the second one usally requires DC voltage supply. The simplest circuit ensuring the AC-DC conversion is the bridge rectifier followed by a filtering capacitance. Nevertheless, this circuit is not optimal for harvesters with a low electromechanical coupling coefficient. Some nonlinear circuits have thus been proposed in the literature as the parallel SSHI (Synchronized Switch Harvesting on Inductor) [4], the series SSHI [5, 6] and the SECE (Synchronized Electric Charge Extraction) [7]. All these pioneer circuits are based on a technique initially developped by Richard et al. for vibration damping [8]. For a comparison review, the reader may refers to the work of Guyomar et al. [9]. Some more complex circuits have also been introduced since then. However, the pioneering circuits mentioned above are still relevant today because 
they offer a simple and effective approach [10,11].

However, these interface circuits have mostly been analysed on linear harvesters on which they show good results. Still few articles propose to study their influence on bistable harvesters. Among them Liu et al. focused on the OSECE circuit, Chen et al. on the series SSHI circuit and Alari et al. on the parallel SSHI circuit [12-14]. Nevertheless, no study has yet proposed any analytical model for a bistable harvester coupled to an AC-DC interface circuit.

The objective of this work is therefore to develop the first analytical model able to predict the frequency response of the bistable harvester coupled to one of them: the SECE circuit. This particular AC-DC circuit has been chosen for two main reasons: (i) it shows promising results on low coupled linear harvesters as the mean power extracted is multiplied by four compared to the use of a simple bridge rectifier [7]; (ii) it allows a decoupling between the harvester and the load, thereby removing the need of adjusting the load impedance to maximize the power harvested. The construction of this mathematical model will be introduced in the next section and then, experimentally validated in the following section. The model will then be used to evaluate the contribution of SECE circuit on bistable harvester by comparing it to a bistable harvester directly connected to the load.

\section{Analytical model}

This section presents the construction of the mathematical model predicting the frequency response of the bistable harvester (with piezoelectric conversion) coupled to the SECE circuit. It will allow to isolate all the possible steady-state behaviors thanks to the harmonic balance method including the subharmonic behaviors for which the mass oscillates with a frequency lower than the excitation ( $n$ times lower for subharmonic $n$ ). Theese particular behaviors have indeed been highlighted for their capability to enhance the global bandwidth of the bistable harvester (especially subharmonic 3) [15, 16]. The mathematical model will also include two criteria to characterize the different behaviors: their stability to small disturbances and their stability robustness. The latest criterion has been used by the authors in a previous study and gives the sensitivity of high orbits (inter-well behaviors) to non-small disturbances [16]. The more robust the high orbit, the less sensitive to external disturbances and therefore the easier to maintain over time without falling on a low orbit. A threshold will be experimentally defined for this criterion above which the high orbits will be considered as robust enough to be used for energy harvesting in real conditions.

The harvester principle and the schematics of the electrical circuit are illustrated in Figure 1. The mechanical part is a one degree of freedom bistable oscillator com- posed by only one piezoelectric converter in order to facilitate the assembly process of the prototype. Contrary to the buckled beam bistable harvester introduced by Liu et al. [17], the piezoelectric converter is here located on the side of the oscillator rather than along the moving beams. This choice has been preferred to reduce its displacement that can damage the electrical connections while its deformations remain the same. The beams on either side of the mass are doubled to remove one unwanted degree of freedom: the rotation of the mass around the axis perpendicular to the plane of the diagram.

Figure 1 also depicts, on a time signal, their expected behavior when the mass is on a high orbit. The variable $x$ corresponds to the position of the mass with respect to the frame, $\pm x_{0}$ its two stable positions, $v$ is the voltage across the piezoelectric converter, $I$ its output current, $R$ represents the load resistance and $v_{D C}$ the voltage across its terminals. The different steps are numbered on this time signal from one to eight: in step 1 the piezoelectric converter is in open circuit (the switches $S_{1}$ and $S_{2}$ are open: $I=0$ ). The voltage therefore follows the evolution of the mass displacement until it reaches a maximum (the piezoelectric converter is charged with energy). Once this maximum of displacement and voltage has been reached, the switch $S_{1}$ is controlled to close (step 2). The piezoelectric converter then discharges its energy into the inductance $L_{1}$. During this phase, the diode $D_{1}$ is blocked which leads to a decoupling between the converter and the smoothing capacitor $C_{1}$. Once all the energy has been discharged $(v=0)$, the switch $S_{1}$ is controlled to open (step 3). The piezoelectric converter passes to open circuit while the inductance $L_{1}$ discharges its energy (by continuity of the current in the inductance) in the smoothing capacitor (the diode $D_{1}$ becomes conductive). The voltage of the piezoelectric converter then follows the evolution of the mass displacement until it reaches a minimum: the piezoelectric converter is charged with energy with a reverse polarity. Once this minimum displacement and voltage is reached, the switch $S_{2}$ is controlled to close (step 4). The piezoelectric converter discharges into the inductance $L_{2}$ then the switch $S_{2}$ is controlled to open (step 5). The converter passes to open circuit while the inductance $L_{2}$ discharges its energy (by continuity of current) in the smoothing capacitor $C_{2}$ and so on.

There are four different phases per period of mass displacement for which the energy is extracted from the piezoelectric converter to the inductance $L_{1}$ or $L_{2}$ : between steps 1 and 2, 3 and 4, 5 and 6, and 7 and 8. Each of these extractions starts when the electrical energy stored in the piezoelectric converter in open circuit reaches a maximum. Indeed, these extractions start when the piezoelectric converter reaches a maximum of compression (steps 1 and 5) or a maximum of elongation (steps 3 and 7). The energy extracted is then stored in the smoothing capacitors $C_{1}$ and $C_{2}$ which continuously 


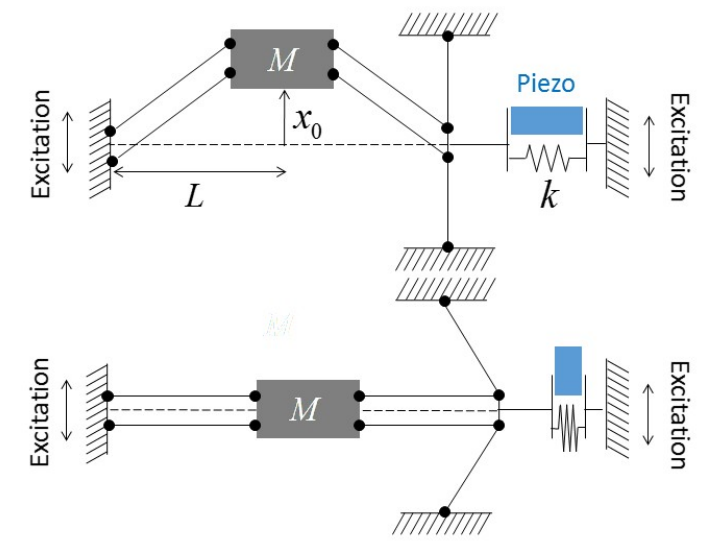

Electrical part

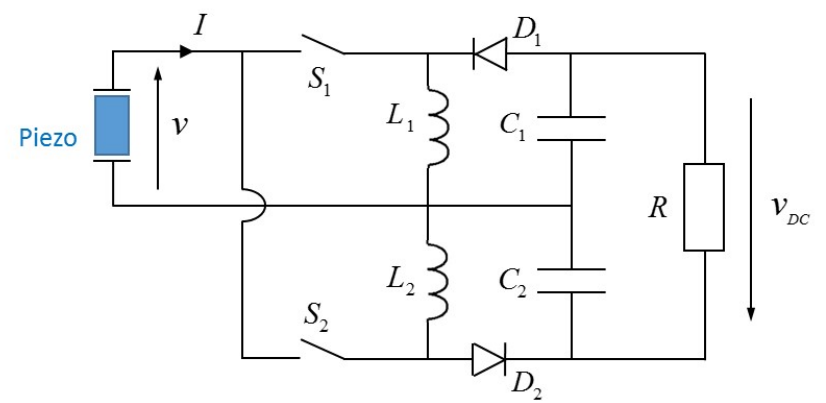

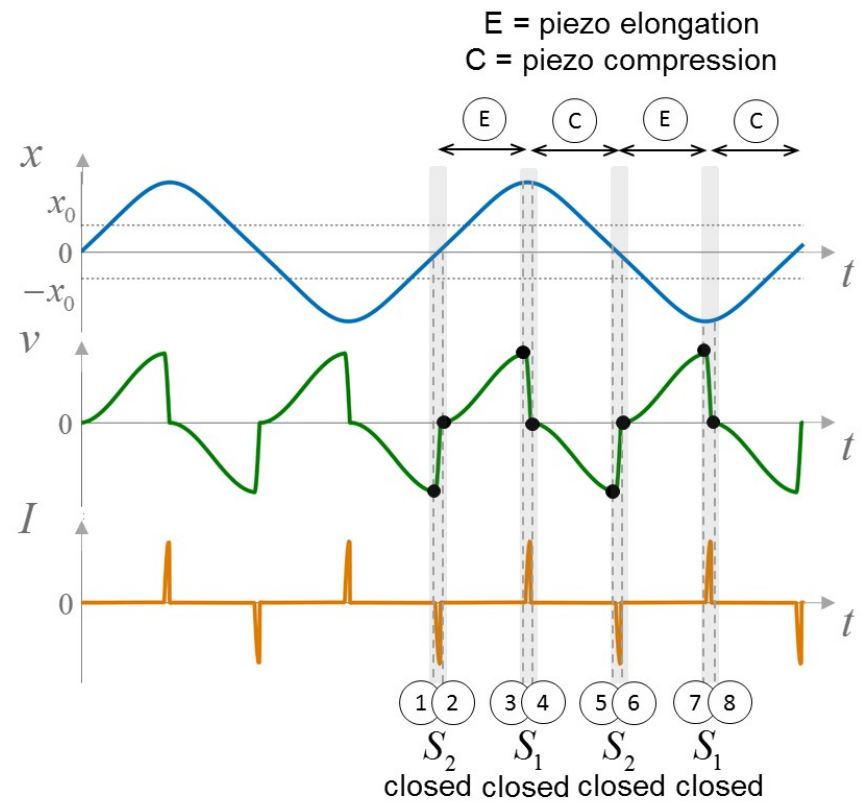

$E=$ piezo elongation

= piezo compression

Figure 1: Bistable harvester and SECE circuit schematics with their expected behavior. The bistable harvester is illustrated in two different equilibrium positions: one of its two stable positions $x= \pm x_{0}$ and its unstable position $x=0$.

discharge into the load resistance. The value of their capacitance is chosen so that the $R C$ circuit period is significantly larger than the mass displacement period. The voltage $v_{D C}$ can therefore be considered as constant over time. The SECE circuit introduced in Figure 1 is slightly different from the common SECE circuit that can be found in literature. It allows a better adaptation to low voltages because the quantity of diodes is reduced removing the bridge rectifier. Their behaviors remain however identical.

\section{II.1. Formulation}

This study focuses on the common harmonic 1 behavior and subharmonic behaviors for which the mass oscillates at a frequency $n$ times lower than the excitation (subharmonic $n$ ). Examples of these behaviors are presented in Figure 4. The general form of the mass position $x$ over time is therefore written as a Fourier series of order $N$ with a fundamental angular frequency equal to $\omega / n$ :

$$
x(t)=a_{0}+\sum_{k=1}^{N}\left\{a_{k} \cos \left(k \frac{\omega}{n} t\right)+b_{k} \sin \left(k \frac{\omega}{n} t\right)\right\}
$$

The variable $a_{0}$ represents the mean value of the mass position. For low orbits (intra-well oscillation), $a_{0} \approx$ $\pm x_{0}$ with $\pm x_{0}$ the two stable positions of the bistable oscillator and for high orbits (inter-well oscillation), $a_{0}=$ 0 . The mechanical part of the harvester is driven by the following equation:

$$
\begin{aligned}
& M(\ddot{x}+A \cos (\omega t)) \\
& =-2 k \underbrace{\left(2 L-2 \sqrt{l^{2}-x^{2}}\right)}_{\text {piezo length variation }} \frac{x}{l}-\mu \dot{x}-2 \alpha v \frac{x}{l}
\end{aligned}
$$

With $M$ the inertial mass, $L$ the horizontal distance separating the mass from the frame, $l$ the length of each rigid four beams, $k$ and $\mu$ the stiffness and the damping coefficient of the oscillator, $\alpha$ and $v$ the force factor and the voltage of the piezoelectric converter. The constants $A$ and $\omega$ represent the magnitude and the angular frequency of the vibration source (sinusoidal acceleration). The term $\alpha v$ reflects the influence of the piezoelectric converter on the mechanical part. This study will focus on low buckling bistable harvesters which can experience high orbit oscillations on a wide bandwidth for low excitations [3]. Hence, $x$ is supposed to be small compared to $L$. Then, the stiffness term of Equation (2) can 
be simplified to:

$$
\begin{aligned}
& -2 k\left(2 L-2 \sqrt{l^{2}-x^{2}}\right) \frac{x}{l} \\
& =-2 k\left(2 L-2 \sqrt{L^{2}+x_{0}^{2}-x^{2}}\right) \frac{x}{\sqrt{L^{2}+x_{0}^{2}}} \\
& =-2 k\left(2-2 \sqrt{1+\frac{x_{0}^{2}-x^{2}}{L^{2}}}\right) \frac{x}{\sqrt{1+x_{0}^{2} / L^{2}}} \\
& =-2 k x\left(-\frac{x_{0}^{2}-x^{2}}{L^{2}}+o\left(\frac{x^{2}}{L^{2}}\right)\right)\left(1-\frac{x^{2}}{2 L^{2}}+o\left(\frac{x^{2}}{L^{2}}\right)\right) \\
& =-2 k x\left(-\frac{x_{0}^{2}-x^{2}}{L^{2}}+o\left(\frac{x^{2}}{L^{2}}\right)\right)
\end{aligned}
$$

The mechanical part of low buckling bistable harvester is therefore modeled by the following Duffing-type equation:

$$
M(\ddot{x}+A \cos (\omega t))=\frac{2 k}{L^{2}}\left(x_{0}^{2}-x^{2}\right) x-\mu \dot{x}-2 \alpha v \frac{x}{L}
$$

The SECE circuit must now be equated to determine a new relationship between $x$ and $v$ which will allow to solve the system. The behavior law of the SECE circuit is defined in a piecewise fashion: its expression changes discontinuously as a function of the diodes states (conductive or blocked). However, the system will be analytically solved with the harmonic balance method which requires non-piecewise expressions valid for every working point. The solution chosen to address this problem is to model the steady-state behaviors considering that the voltage signal can be seen as a sum of two signals: the voltage in open circuit and a square signal. This decomposition is shown in Figure 2 and can be written as follows:

$$
v_{\text {steady-state }}=\frac{v_{\text {open circuit }}}{\text { depends on } x}+\frac{v_{\text {square }}}{\text { depends on } x}
$$

This expression gives a new relationship between $x$ and $v$ which can be used to analytically solve the system with the harmonic balance method. Indeed $v_{\text {open circuit }}$ has a non-piecewise expression and $v_{\text {square }}$ can be approached by a truncated Fourier series.

The square signal fronts reflect the extractions of energy from the piezoelectric converter which occur when the mass position is zero (the piezo is fully compressed) or when the mass position reaches a maximum or a minimum (the piezo is fully elongated). It has to be noted that, for a bistable harvester in open circuit, the maximum voltage reached by the piezoelectric converter is not necessary equal to its minimum voltage. The first one appears when the elongation of the converter is maximum (i.e., when the mass position reach an extremum) and the second one when the compression is maximum (i.e., when the mass position is zero). This elongation and this compression are directly linked to the distance between the mass position and its stable position $\left|x-x_{0}\right|$ which is not the same when the mass position is zero or when it reaches an extremum.

The piezoelectric converter is equivalent to a current generator (proportional to the speed of its length variation) in parallel with a capacitor. In open circuit, this law becomes:

$$
C_{0} \dot{v}_{\text {open circuit }}=\alpha \frac{\mathrm{d}}{\mathrm{d} t} \underbrace{\left(2 L-2 \sqrt{l^{2}-x^{2}}\right)}_{\text {piezo length variation }}
$$

With $C_{0}$ and $\alpha$ the blocked capacitance and the force factor of the piezoelectric converter. This equation can be approached considering that $l^{2}=L^{2}+x_{0}^{2}$ and that $x$ is small compared to $L$ (bistable oscillator with a small buckling level) to give the following expression:

$$
\begin{aligned}
C_{0} \dot{v}_{\text {open circuit }} & =2 \alpha \frac{x}{L} \dot{x} \\
\Rightarrow v_{\text {open circuit }} & =\frac{\alpha}{C_{0} L}\left(x^{2}-x_{0}^{2}\right)
\end{aligned}
$$

The constant of integration has been chosen to impose a voltage equal to zero when the mass is on a stable position. The square signal can be written as a Fourier series. Its amplitude $\Theta_{\mathrm{S}}$ and its frequency must be equal to the amplitude and the frequency of $v_{\text {open circuit }}$ and its mean value $o_{\mathrm{s}}$ equals, in absolute value, to the mean of $v_{\text {open circuit }}$ (but with an opposite sign):

$$
v_{\text {square }}=o_{\mathrm{s}}+\Theta_{\mathrm{s}} \frac{4}{\pi} \sum_{k=0}^{+\infty} \frac{1}{2 k+1} \sin \left((2 k+1)\left(\frac{2 \omega}{n} t+\phi\right)\right)
$$

with

$$
\begin{aligned}
\Theta_{\mathrm{s}} & =\frac{v_{\max }-v_{\min }}{2} \\
& =\frac{\alpha}{2 C_{0} L}\left(\left(|x|_{\max }{ }^{2}-x_{0}^{2}\right)-\left(|x|_{\min }{ }^{2}-x_{0}^{2}\right)\right) \\
& =\frac{\alpha}{2 C_{0} L}\left(|x|_{\max ^{2}}{ }^{2}-|x|_{\min }{ }^{2}\right)
\end{aligned}
$$

and

$$
\begin{aligned}
o_{\mathrm{s}} & =-\left(v_{\min }+\frac{v_{\max }-v_{\min }}{2}\right) \\
& =-\frac{\alpha}{C_{0} L}\left(|x|_{\min }{ }^{2}-x_{0}^{2}\right)-\frac{\alpha}{2 C_{0} L}\left(|x|_{\max }{ }^{2}-|x|_{\min ^{2}}{ }^{2}\right) \\
& =\frac{\alpha}{2 C_{0} L}\left(2 x_{0}^{2}-|x|_{\max }{ }^{2}-|x|_{\min }{ }^{2}\right)
\end{aligned}
$$

The value of $|x|_{\min }$ and $|x|_{\max }$ depends on the studied orbit as shown in Figure 3. In particular $|x|_{\min }=0$ for high orbits contrary to low orbits.

The phase of the square signal $\phi$ still needs to be determined as a function of $x$. When the mass position reaches a maximum, the square signal must go down and be equal to zero. Hence, when the mass position reaches a maximum, the phase of all the sinus in the Fourier series must be equal to $\pi$. The phase $\phi$ can 
therefore be determined knowing the instants on which the mass position reaches a maximum. To simplify the reasoning, these instants are calculated considering that the mass position can be approached by its fundamental component. The relevance of this assumption is justified by the examples shown in Figure 4 . In this Figure, three different behaviors of the bistable harvester in open circuit are shown: the common harmonic 1 low and high orbits and the subharmonic 3 high orbit. Each example details, for the considered behavior, the mass position over time as well as its fundamental component. They have been obtained numerically solving the system composed by Equations (4) and (8) with the parameter values corresponding to the prototype which will be introduced in the experimental section. The fundamental component of the subharmonic $n$ behavior can be written as:

$$
\begin{aligned}
x_{\text {fundamental }}(t) & =a_{1} \cos \left(\frac{\omega}{n} t\right)+b_{1} \sin \left(\frac{\omega}{n} t\right) \\
& =\sqrt{a_{1}^{2}+b_{1}^{2}} \sin \left(\frac{\omega}{n} t+\psi\right)
\end{aligned}
$$

with

$$
\psi=\tan ^{-1}\left(\frac{a_{1}}{b_{1}}\right)+\pi\left(\text { if } b_{1}<0\right)
$$

The instant on which the mass reaches a maximum is called $t_{1}$. The mass displacement phase in $t_{1}$ is therefore equal to $\pi / 2$ :

$$
\begin{aligned}
\frac{\omega}{n} t_{1}+\psi & =\frac{\pi}{2} \bmod [2 \pi] \\
\Rightarrow t_{1} & =\left(\frac{\pi}{2}-\psi\right) \frac{n}{\omega} \bmod \left[2 \pi \frac{n}{\omega}\right]
\end{aligned}
$$

The phase of all the square signal sinus terms must then be equal to $\pi$ in $t_{1}$. In particular, the phase of the fundamental one yields:

$$
\begin{array}{rlr}
\frac{2 \omega}{n} t_{1}+\phi & =\pi \quad \bmod [2 \pi] \\
\Rightarrow \frac{2 \omega}{n}\left(\frac{\pi}{2}-\psi\right) \frac{n}{\omega}+\phi & =\pi \quad \bmod [2 \pi] \\
\Rightarrow \phi & =2 \psi & \bmod [2 \pi]
\end{array}
$$

In conclusion, the steady-state behavior of the bistable harvester coupled to SECE circuit is governed by the combination of Equation (4) and Equation (5) giving:

$$
\left\{\begin{array}{l}
\ddot{x}+\frac{\omega_{0}^{2}}{2}\left(\frac{x^{2}}{x_{0}^{2}}-1\right) x+\frac{\omega_{0}}{Q} \dot{x}+\frac{2 \alpha}{M L} x v=-A \cos (\omega t) \\
v=\frac{\alpha}{C_{0} L}\left(x^{2}-x_{0}^{2}\right) \\
\quad+o_{\mathrm{s}}+\Theta_{\mathrm{s}} \frac{4}{\pi} \sum_{k=0}^{N_{\mathrm{bis}}} \frac{1}{2 k+1} \sin \left((2 k+1)\left(\frac{2 \omega}{n} t+2 \psi\right)\right)
\end{array}\right.
$$

with

$$
\left\{\begin{array}{l}
\omega_{0}=\frac{x_{0}}{L} \sqrt{\frac{4 k}{M}} \\
Q=\frac{x_{0}}{L} \frac{\sqrt{4 k M}}{\mu}
\end{array}\right.
$$

The constants $\omega_{0}$ and $Q$ correspond to the natural angular frequency and the mechanical quality factor of the equivalent linear oscillator obtained for a small excitation of the bistable oscillator inducing small displacements around one of its two stable positions $\left(x=x_{0}+\Delta x\right.$ with $\Delta x<<x_{0}$ ) [17]. The general solution sought for this system is the subharmonic $n$ behavior written in the form of a Fourier series of order $N$ :

$$
x(t)=a_{0}+\sum_{k=1}^{N}\left\{a_{k} \cos \left(k \frac{\omega}{n} t\right)+b_{k} \sin \left(k \frac{\omega}{n} t\right)\right\}
$$

In this case, the constants of the square signal are given by the following equations:

$$
\left\{\begin{array}{l}
\Theta_{\mathrm{s}}=\frac{\alpha}{2 C_{0} L}\left(|x|_{\max }^{2}-|x|_{\text {min }}^{2}\right) \\
o_{\mathrm{s}}=\frac{\alpha}{2 C_{0} L}\left(2 x_{0}^{2}-|x|_{\max }^{2}-|x|_{\text {min }}^{2}\right) \\
\psi=\tan ^{-1}\left(\frac{a_{1}}{b_{1}}\right)+\pi\left(\text { if } b_{1}<0\right) \\
|x|_{\max }=\left|a_{0}\right|+\sqrt{a_{1}^{2}+b_{1}^{2}} \\
|x|_{\min }=\left|a_{0}\right|-\sqrt{a_{1}^{2}+b_{1}^{2}} \quad \text { (low orbit) } \\
|x|_{\min }=0 \quad \text { (high orbit) }
\end{array}\right.
$$

Here again, $|x|_{\min }$ and $|x|_{\max }$ are calculated considering that the mass displacement can be approached by its fundamental component in order to simplify their expressions. The relevance of this assumption can be checked on the examples shown in Figure 4. Similarly to the Fourier series of the mass displacement, the Fourier series of the square signal is truncated at a particular

\section{Piezo in open circuit}

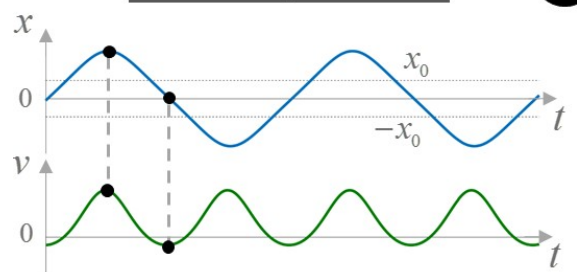

Discontinuity

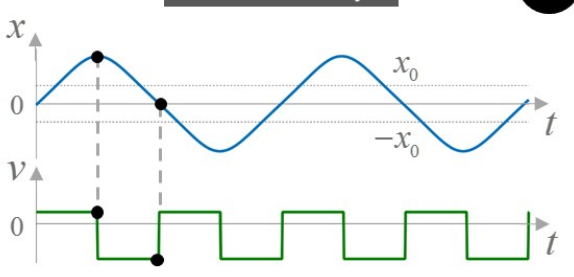

Steady-state SECE

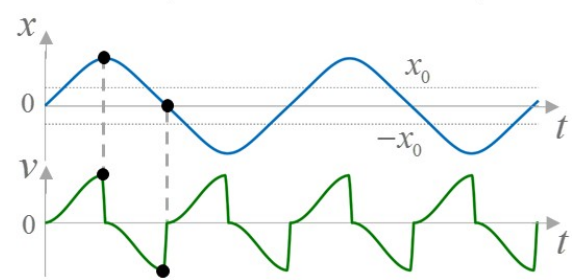

Figure 2: Decomposition of the voltage accross the piezoelectric element for the bistable harvester coupled to SECE circuit in steady state. 


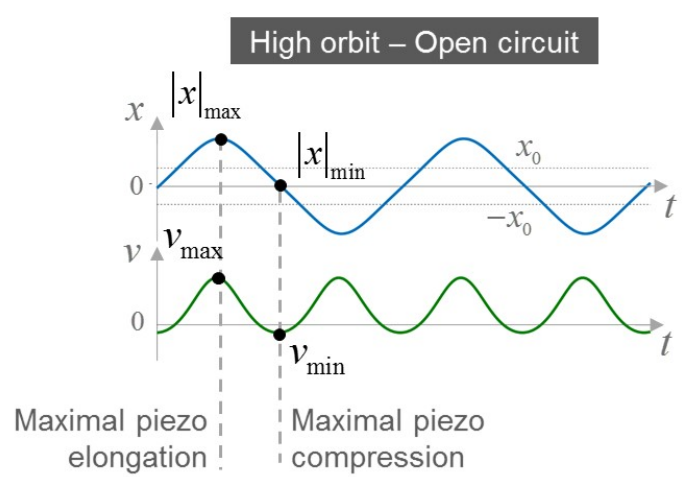

Figure 3: Values of $|x|_{\min },|x|_{\max }, v_{\min }$ and $v_{\max }$ according to the studied orbit.
Low orbit - Open circuit

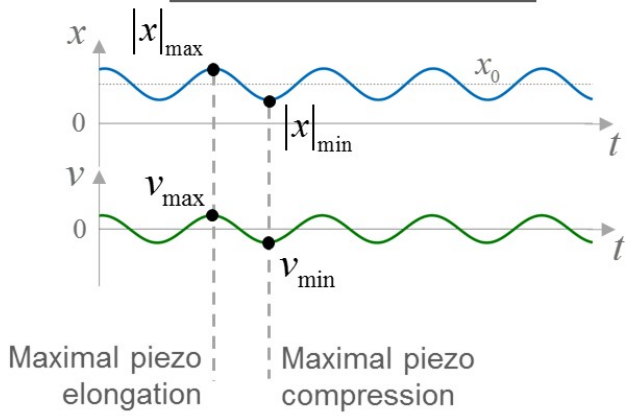

order $N_{\text {bis. }}$ For the mass displacement, all the angular frequencies above $N \omega / n$ are neglected. Hence, all the angular frequencies above $N \omega / n$ are also neglected in the square signal:

$$
\begin{aligned}
\left(2 N_{\text {bis }}+1\right) \frac{2 \omega}{n} & =N \frac{\omega}{n} \\
\Rightarrow N & =4 N_{\text {bis }}+2
\end{aligned}
$$

The two equations of the law governing the behavior of the bistable harvester coupled to the SECE circuit (Equation (19)) can be combined in a single equation which only depends on the unknown $x$ (mass displacement). An approximated solution can then be found for this single equation thanks to the harmonic balance method. The solution has to be searched in the form of a truncated Fourier series which is detailed in Equation (21). For more details on the application of this method, the reader may refer to the similar analytic analyses used for a bistable harvester (with electromagnetic coupling) connected to a simple resistance [16]. Each approximated solution that comes up with this method is defined by a unique set $\left(a_{s 0}, a_{s k}, b_{s k}\right)$ with $k \in \llbracket 1, N \rrbracket$. They all correspond to possible steady-state subharmonic $n$ behaviors of the bistable harvester coupled to SECE circuit for an excitation $A \cos (\omega t)$ :

$$
\left\{\begin{array}{l}
x_{s}(t)=a_{s 0}+\sum_{k=1}^{N}\left\{a_{s k} \cos \left(k \frac{\omega}{n} t\right)+b_{s k} \sin \left(k \frac{\omega}{n} t\right)\right\} \\
v_{\mathcal{S}}(t)=\frac{\alpha}{C_{0} L}\left(x_{s}(t)^{2}-x_{0}^{2}\right) \\
+o_{\mathrm{S}}+\Theta_{\mathrm{s}} \frac{4}{\pi} \sum_{k=0}^{N_{\mathrm{bis}}} \frac{1}{2 k+1} \sin \left((2 k+1)\left(\frac{2 \omega}{n} t+2 \psi\right)\right)
\end{array}\right.
$$

It has to be noted that several solutions may coexist for a given excitation due to the nonlinear nature of the governing law. The harvester will stabilize on one of them according to its initial conditions (initial position, initial speed and initial voltage). To obtained the complete frequency response, the approximated solutions of Equation (19) have to be determined in this way for different angular frequencies of the excitation $\omega$ and for different kind of steady-state behaviors (i.e., different values of $n$ ).

\section{II.2. Stability to small disturbances}

The stability of the different steady-state behaviors $x_{s}$ are then analyzed thanks to the common study of stability to small disturbances. Thus, a small disturbance $z$ is added to the mass displacement in the form of a pulse at the instant $t_{0}\left(z\left(t_{0}\right)\right.$ and $\dot{z}\left(t_{0}\right)$ are different from zero). The solution $x_{s}$ is stable to small disturbances if and only if $z$ tends toward zero:

$$
\begin{gathered}
x(t)=x_{s}(t)+z(t) \quad \text { with }\|z(t)\|<<\left\|x_{s}(t)\right\| \\
\text { and pulse }=\left(\begin{array}{c}
z\left(t_{0}\right) \\
\dot{z}\left(t_{0}\right)
\end{array}\right)
\end{gathered}
$$

The Equation (26) is injected in Equation (19) governing the behavior of the bistable harvester coupled to SECE circuit and linearized for small $z$ to give the differential equation governing the disturbance:

$$
\begin{aligned}
\left(\begin{array}{c}
\dot{z}(t) \\
\ddot{z}(t)
\end{array}\right) & =\left[\begin{array}{cc}
0 & 1 \\
m & -\frac{\omega_{0}}{Q}
\end{array}\right]\left(\begin{array}{l}
z(t) \\
\dot{z}(t)
\end{array}\right) \\
\Leftrightarrow & \left(\begin{array}{l}
\dot{z}(t) \\
\ddot{z}(t)
\end{array}\right)=\underline{\underline{M}}(t)\left(\begin{array}{l}
z(t) \\
\dot{z}(t)
\end{array}\right)
\end{aligned}
$$

with

$$
\begin{aligned}
& m=\frac{\omega_{0}^{2}}{2}+\frac{2 \alpha^{2} x_{0}^{2}}{C_{0} M L^{2}}-\left(\frac{3 \omega_{0}^{2}}{2 x_{0}^{2}}+\frac{6 \alpha^{2}}{C_{0} M L^{2}}\right) x_{s}(t)^{2}-\frac{2 \alpha}{M L}\{ \\
& \left.o_{\mathrm{s}}+\Theta_{\mathrm{s}} \frac{4}{\pi} \sum_{k=0}^{N_{\text {bis }}} \frac{1}{2 k+1} \sin \left((2 k+1)\left(\frac{2 \omega}{n} t+2 \psi\right)\right)\right\}
\end{aligned}
$$

From there, the stability to small disturbances can be determined with the Floquet theory and the Lyapunov exponents. For more details on the application of this method, the reader may refer to the similar analytic analyses used for a bistable harvester (with electromagnetic coupling) connected to a simple resistance [16]. The stability of the different steady-state behaviors $x_{S}$ can finally be determined with the eigenvalues $\left(\beta_{1}, \beta_{2}\right)$ of the resolvent matrix $\underline{\underline{R}}(2 T, 0)$, obtained after two numerical integration of Equation (28):

$$
\underline{\underline{R}}(2 T, 0)=\left[\left(\begin{array}{l}
z_{1}(2 T) \\
\dot{z}_{1}(2 T)
\end{array}\right)\left(\begin{array}{l}
z_{2}(2 T) \\
z_{2}(2 T)
\end{array}\right)\right]
$$



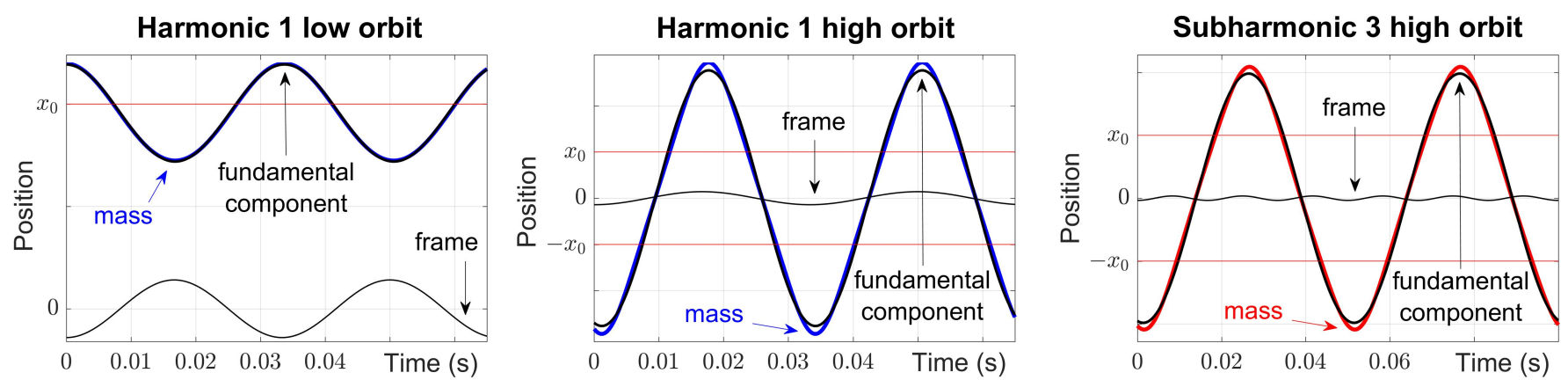

Figure 4: Difference between the mass displacement signal (polychromatic) and the signal of its fundamental component. This numerical study has been done with an excitation of $30 \mathrm{~Hz}$ for harmonic 1 high and low orbit and with an excitation of $60 \mathrm{~Hz}$ for subharmonic 3 high orbit.

with

$$
\left\{\begin{array}{l}
\left(\begin{array}{l}
z_{1}(0) \\
z_{1}(0)
\end{array}\right)=\left(\begin{array}{l}
1 \\
0
\end{array}\right) \underset{\text { numerical integration of }}{\stackrel{\text { Equation (28) from } 0 \text { to } 2 T}{\longrightarrow}}\left(\begin{array}{l}
z_{1}(2 T) \\
z_{1}(2 T)
\end{array}\right) \\
\left(\begin{array}{l}
z_{2}(0) \\
z_{2}(0)
\end{array}\right)=\left(\begin{array}{l}
0 \\
1
\end{array}\right) \underset{\text { numerical intégration }}{\stackrel{\text { Equation (28) from } 0 \text { to } 2 T}{\longrightarrow}}\left(\begin{array}{l}
z_{2}(2 T) \\
z_{2}(2 T)
\end{array}\right)
\end{array}\right.
$$

and

$$
x_{s} \text { stable } \Leftrightarrow\left\{\begin{array}{l}
\left|\beta_{1}\right|<1 \\
\left|\beta_{2}\right|<1
\end{array}\right.
$$

\section{II.3. Stability robustness}

It is now possible to determine the stability to small disturbances of the different behaviors $x_{s}$ of the bistable harvester coupled to SECE circuit. However, the behaviors which are stable with this criterion, do not necessarily have the same sensitivity to bigger disturbances. The latter can indeed appear in real condition applications or can be brought by the mechanical defaults of the prototype. In particular, these bigger disturbances may cause a destabilization of high orbits which would collapse on a low orbit. The different high orbits do not have the same sensitivity to bigger disturbances. A criterion taking this aspect into account have been used in a previous study: the stability robustness [16]. The more robust the high orbit, the less sensitive to bigger disturbances and therefore the easier to maintain over time without falling on a low orbit. A threshold can then be defined for this criterion above which the high orbits will be considered as robust enough to be used for energy harvesting in real conditions.

A disturbance $z$ is added to the stable high orbits in the form of a speed pulse $\Lambda$ at the instant $t_{0}$. This disturbance is not considered anymore as small compared to the mass displacement:

$$
x(t)=x_{s}(t)+z(t) \text { and } \text { pulse }=\left(\begin{array}{c}
z\left(t_{0}\right) \\
\dot{z}\left(t_{0}\right)
\end{array}\right)=\left(\begin{array}{c}
0 \\
\Lambda
\end{array}\right)
$$

If the speed pulse $\Lambda$ is small, we come back to the small disturbance analysis therefore $z$ will tends toward zero because the studied high orbit is stable. If we increase the speed pulse $\Lambda$ (in absolute value), this high orbit will end up being destabilized and fall down on a low orbit. The minimum speed pulse $\Lambda_{\text {min }}$ which destabilizes the high orbit will characterize the stability robustness. More particularly, this criterion is defined by the ratio between the minimum energy that this pulse must have to destabilize the high orbit and the average amount of energy provided by the excitation during one period (normative value):

$$
\text { Robustness }=\frac{\frac{1}{2} M \Lambda_{\text {min }}^{2}}{E_{\text {excitation }}}
$$

with

$$
E_{\text {excitation }}=-\frac{1}{n} \int_{0}^{T} M A \cos (\omega t) \dot{x_{s}} d t
$$

Thus, the objective is to determine $\Lambda_{\min }$. Equation (33) is injected in Equation (19) governing the behavior of the bistable harvester coupled to SECE circuit to give the differential equation governing the disturbance:

$$
\begin{aligned}
\left(\begin{array}{l}
\dot{z}(t) \\
\ddot{z}(t)
\end{array}\right) & =\underbrace{\underline{\underline{M}(t)\left(\begin{array}{l}
z(t) \\
\dot{z}(t)
\end{array}\right)}}_{\text {linear term }} \\
& +\underbrace{\left(-\left(\frac{\omega_{0}^{2}}{2 x_{0}^{2}}+\frac{2 \alpha^{2}}{C_{0} M L^{2}}\right)\left(3 x_{s}(t) z(t)^{2}+z(t)^{3}\right)\right)}_{\text {nonlinear term }}
\end{aligned}
$$

The matrix $\underline{M}(t)$ is the same as the one introduced in the small disturbance analyses. From this equation, it is possible, thanks to a reductio ad absurdum, to determine the minimal speed pulse $\Lambda_{\min }$ which will cause the divergence of the disturbance $z$ :

$$
\Lambda_{\min }=\frac{\delta_{0}-B \delta_{0}^{2}-D \delta_{0}^{3}}{[\underline{\underline{C}}]_{(1,2)}}
$$


with

$$
\left\{\begin{array}{l}
\delta_{0}=\frac{-B+\sqrt{B^{2}+3 D}}{3 D} \\
B=\left(\frac{3 \omega_{0}^{2}}{2 x_{0}^{2}}+\frac{6 \alpha^{2}}{C_{0} M L^{2}}\right)[\underline{\underline{C}}]_{(1,2)} \max _{t_{0} \in[0, T]}|\Psi| \\
D=\frac{1}{2 \lambda}\left(\frac{\omega_{0}^{2}}{2 x_{0}^{2}}+\frac{2 \alpha^{2}}{C_{0} M L^{2}}\right)[\underline{\underline{C}}]_{(1,2)} \\
{[\underline{\underline{C}}]_{(1,2)}=\max _{\substack{t_{0} \in[0, T] \\
t \in\left[t_{0}, t_{0}+2 T\right]}}\left|e^{\lambda\left(t-t_{0}\right)}\left[\underline{\underline{R}}\left(t, t_{0}\right)\right]_{(1,2)}\right|}
\end{array}\right.
$$

and

$$
\left\{\begin{aligned}
\lambda & =-\frac{\max \left(\left(\ln \left(\beta_{1}\right), \ln \left(\beta_{2}\right)\right)\right.}{2 T} \\
\Psi & =\sum_{k=1}^{N}\left\{\frac{a_{s k}}{\lambda^{2}+\omega_{k}^{2}}\left(\lambda \cos \left(\omega_{k} t_{0}\right)-\omega_{k} \sin \left(\omega_{k} t_{0}\right)\right)\right. \\
& \left.+\sum_{k=1}^{N} \frac{b_{s k}}{\lambda^{2}+\omega_{k}^{2}}\left(\lambda \sin \left(\omega_{k} t_{0}\right)+\omega_{k} \cos \left(\omega_{k} t_{0}\right)\right)\right\} \\
T & =2 \pi n / \omega \\
\omega_{k} & =k \omega / n
\end{aligned}\right.
$$

The methodology that leads to this result is entirely detailed in the similar analytic analyses that have been conducted for a bistable harvester (with electromagnetic coupling) connected to a simple resistance [16]. The reader may refer to this study for more details. The influence of the SECE circuit, which comes from the square signal, only appears in the expression of the matrix $\underline{M}(t)$. Hence, the influence of the SECE circuit on the calculation of the minimal speed pulse $\Lambda_{\min }$ appears in the calculation of the coefficient $[\underline{\underline{C}}]_{(1,2)}$ and in the calculation of the coefficient $\lambda$ both linked to the resolvent matrix $\underline{\underline{R}}\left(t, t_{0}\right)$ of $\underline{\underline{M}}(t)$.

The mathematical model of the bistable harvester coupled to SECE circuit is now complete. It allows to predict the frequency response of the harvester detailing all the reachable steady-state subharmonic behaviors. This model also includes two characterization criteria for the different behaviors: their stability to small disturbances and their stability robustness (i.e., their robustness to external disturbances in real conditions). An experimental analysis has been conducted to validate this model. The next section introduces this experimental analysis. The analytic and experimental results are gathered in Figure 9 in the following section.

\section{Experimental analysis}

This section introduces the experimental set up and the prototype developed to measure the influence of the SECE circuit on the bistable harvester and to validate the mathematical model presented in the previous section. Pictures are shown in Figure 5.
The combination of the frame, the mass, the steel beams and the converter Piezo harvester forms the bistable harvester, subject of this study. The actuator Piezo buckling modifier is not used in this experiment (shortcircuited). Its stiffness being chosen big compared to Piezo harvester's one, its influence on the harvester behavior is neglected. The beams on either side of the mass are doubled to remove one unwanted degree of freedom: the rotation of the mass around the axis perpendicular to the plane of the diagram. To get the bistable configuration, these beams are buckled thanks to the nut which allow translating the threaded rod in the direction of the mass. The buckling level of the bistable harvester is then adjusted with this nut and kept low thus validating the assumption made to establish the mathematical model illustrated in Figure 1.

From a technical point of view, the prototype was obtained by cutting a single block of APX4 steel by electrical discharge machining in order to reveal the mass, the beams and the frame. The two piezoelectric converter are then added. The pivoting links, visible in Figure 1,

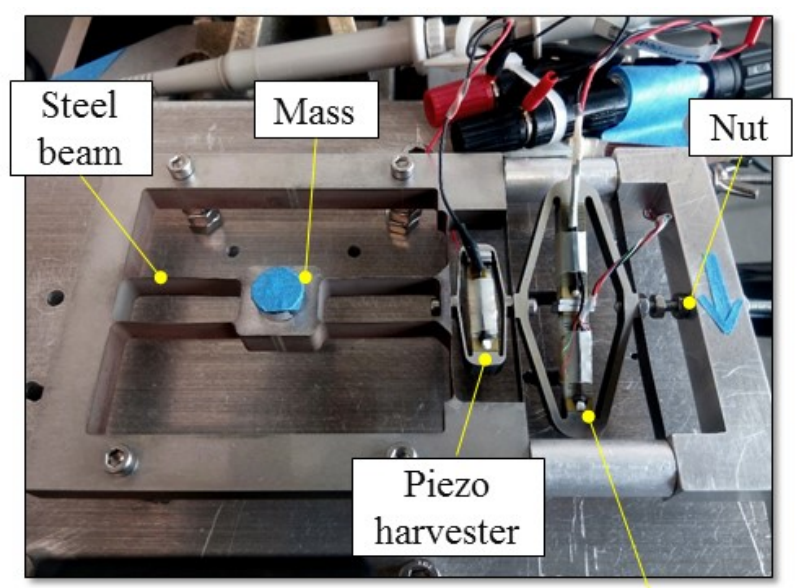

(Piezo buckling modifier)

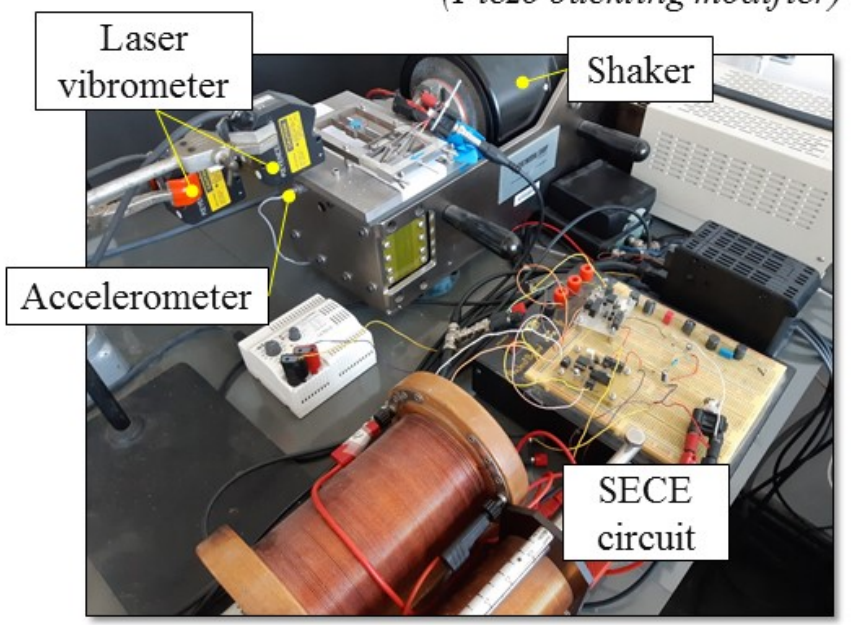

Figure 5: Prototype of the bistable harvester (buckled beams) with a piezoelectric converter ("Piezo harvester") connected to a SECE circuit. The actuator named "Piezo buckling modifier" is not used here and is short-circuited. Its influence on the behavior of the harvester is neglected (its stiffness is much larger than the "Piezo harvester"'s one). The time step imit of the acquisition card used (Dspace: $100 \mu \mathrm{s}$ ) implies high inductance values. The two coils used are therefore relatively large in order to keep the electrical losses acceptable. The optimization of these coils is not part of this study. 
are here ensured by the flexibility of the thin steel beams (flexible bonds). These flexible bonds have a negligible effect on the stiffness and damping of the bistable harvester and can therefore be modeled as pure pivoting links. Indeed, (i) the potential energy stored in these flexible bonds is small compared to the potential energy stored in the Piezo harvester (for low buckling configuration) and (ii) the mechanical losses in these bonds are negligible thanks to the material used (APX4 steel).

The Piezo harvester is a piezoelectric converter from Cedrat Technologies called APA 120S (Amplified Piezoelectric Actuator). The electromechanical coupling factor of these actuators is indeed better than the one of a simple piezoelectric patch glued on a the beams because the 33 mode is used. They are composed by two elements: an elliptical stainless steel shell and a stack of interdigital piezoelectric ceramics (PZT) placed on the main axis of this shell. The elliptical shape acts as a mechanical transformer. It amplifies on the long axes the stress applied on the short axes. On the contrary, it reduces on the long axes the strain applied on the short axes. This shell thus allow to optimize the use of the piezoelectric ceramics which can handle strong stresses but small strains. The main dimensions of this prototype are detailed in Table 1.

The parameters $\left(\omega_{0}, Q, C_{0}, \alpha\right)$ of the bistable harvester have been identified on the prototype following the methodology presented by Badel et al. [18]. This methodology consists on measuring the complex admittance $\underline{Y}$ of the Piezo harvester around the natural frequency of bistable harvester without any external excitation. The measured admittance is then used to adjust the different parameters of the following expression:

$$
\left\{\begin{array}{l}
\underline{Y}=\frac{\underline{I}}{\underline{V}}=j C_{0} \omega\left(1+\frac{k_{m}^{2}}{1-\left(\omega / \omega_{0}\right)^{2}+j \frac{\omega / \omega_{0}}{Q}}\right) \\
k_{m}^{2}=4\left(\frac{x_{0}}{L}\right)^{2} \frac{\alpha^{2}}{M C_{0} \omega_{0}^{2}}
\end{array}\right.
$$

Figure 6 shows this adjustment between the measured admittance and the theoretical admittance. The value of all the parameters of the prototype and of the SECE circuit are summarized in Table 2. The electromechanical coupling factor is given for the equivalent linear oscillator obtained for a small excitation of the bistable harvester inducing small displacements around one of

Table 1: Prototype main dimensions.

\begin{tabular}{lcc}
\hline \hline Elément & Value & Unity \\
\hline Inertial mass weight & 17.3 & $\mathrm{~g}$ \\
Inertial mass dimensions & $16 \times 16 \times 8.0$ & $\mathrm{~mm}^{3}$ \\
APX4 steel block thickness & 8.0 & $\mathrm{~mm}$ \\
Steel beams thickness & 0.070 & $\mathrm{~mm}$ \\
Horizontal beams length & 25 & $\mathrm{~mm}$ \\
Vertical beams length & 17 & $\mathrm{~mm}$ \\
Piezo harvester stiffness & 0.30 & N. $\mu \mathrm{m}^{-1}$ \\
\hline \hline
\end{tabular}

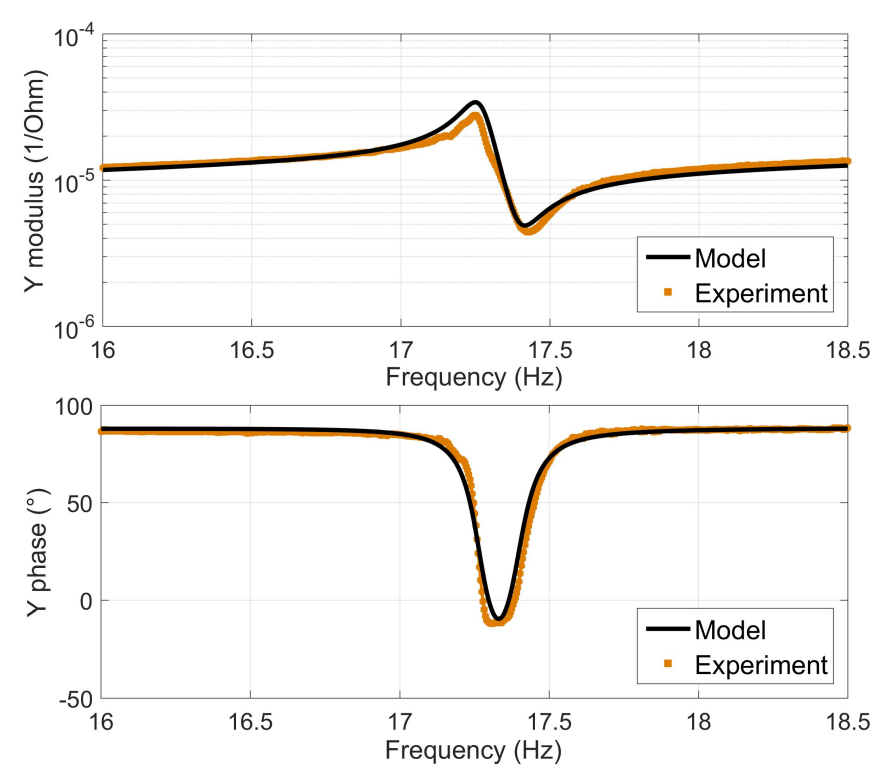

Figure 6: Admittance adjustment in order to identify the parameters $\left(\omega_{0}, Q, C_{0}, \alpha\right)$ of the prototype.

its two stable positions $\left(x=x_{0}+\Delta x\right.$ with $\left.\Delta x<<x_{0}\right)$ [17].

The prototype has been installed on a shaker which provides a sinusoidal acceleration of constant amplitude set at $2.5 \mathrm{~m} . \mathrm{s}^{-2}$. The position of the mass and the position of the frame have been measured thanks to two laser displacement sensors. The voltage at the terminals of the Piezo harvester and at the terminals of the resistance load have been measured with two differential voltage probes. The switches of the SECE circuit have been controlled to close on extrema of the Piezo harvester voltage (on maxima for switch $S_{1}$ and on minima for switch $S_{2}$ ). They have been controlled to open when the Piezo harvester voltage reaches zero. The frequency response of the bistable harvester coupled to the SECE circuit has then been explored analyzing the frequency ranges of the different steady-state behaviors with the following method: (i) the excitation is set at a frequency where the studied behavior exists; (ii) force pulses are applied to the mass imposing square voltage pulses to the Piezo harvester $(20 \mathrm{~ms}$ and $30 \mathrm{~V})$ until the studied

Table 2: Parameters of the bistable harveter prototype and SECE circuit.

\begin{tabular}{lccc}
\hline \hline Parameter & Symbol & Value & Unit \\
\hline Stable positions & $\pm x_{0}$ & \pm 0.50 & $\mathrm{~mm}$ \\
Mass-frame distance & $L$ & 25 & $\mathrm{~mm}$ \\
Inertial mass & $M$ & 17.3 & $\mathrm{~g}$ \\
Natural angular frequency & $\omega_{0}$ & 121 & $\mathrm{rad}^{-1}$ \\
Mechanical quality factor & $Q$ & 87 & - \\
Excitation magnitude & $A$ & 2.5 & $\mathrm{~m} . \mathrm{s}^{-2}$ \\
Excitation frequency & $\omega$ & $15-85$ & $\mathrm{~Hz}$ \\
Piezo harvester force factor & $\alpha$ & 0.068 & $\mathrm{~N} . \mathrm{V}^{-1}$ \\
Piezo harvester blocked capacity & $C_{0}$ & 1.05 & $\mu \mathrm{F}$ \\
Coils inductance & $L_{1}-L_{2}$ & 500 & $\mathrm{mH}$ \\
Smoothing capacitors & $C_{1}-C_{2}$ & 1.00 & $\mu \mathrm{F}$ \\
Load resistance & $R$ & 100 & $\mathrm{k} \Omega$ \\
Electromechanical coupling factor & $k_{m}^{2}$ & 2.74 & $\%$ \\
\hline
\end{tabular}


behavior is reached The force pulses indeed destabilize the steady-state behavior and potentially lead to a another steady-state behavior coexisting at this particular frequency; (iii) once the studied behavior is reached, the excitation frequency is slowly and smoothly increased or decreased to explore its frequency range without destabilizing it.

For this study, only the most interesting behaviors for energy harvesting have been investigated: the common harmonic 1 high orbit which proposes the working point with the maximum mean harvested power and the subharmonic 3 high orbit which allows widening the global bandwidth of the bistable harvester since its frequency range appears where the harmonic 1 behavior can not be reached [15]. Figure 7 and 8 show examples of time signals of these two behaviors obtained both experimentally and with the model constructed in the previous section. Experiment and theory are here in good agreement. The square signal, used in the decomposition of the theoretical Piezo harvester voltage, has been approached at the order $N_{\text {bis }}=10$. Hence, the theoretical mass displacement has been sought as a Fourier series truncated at the order $N=42$ as stated in Equation (24).

\section{Results and discussion}

Figure 9 shows both the experimental and the theoretical frequency responses of the bistable harvester coupled to the SECE circuit. These frequency responses focus on the two most interesting behaviors for energy harvesting: harmonic 1 high orbit and subharmonic 3 high orbit [15]. These frequency responses only detail the working points defined as stable with the stability to small disturbances analysis. Four different quantities are drawn to characterize these two orbits as a function of the excitation frequency: (i) the amplitude of the mass oscillations; (ii) the phase of the excitation displacement when the position of the mass reaches a maximum; (iii) the stability robustness; (iv) the mean extracted power (theory and experiment) and the mean harvested power (experiment).

For the theoretical curves, the phase of the excitation displacement when the position of the mass reaches a maximum is calculated as follows. First, to simplify the reasoning, the mass position signal is approached by its fundamental harmonic component. The reader may refer to Figure 4 to check the relevance of this assumption. This fundamental component can be written as:

$$
\begin{aligned}
x_{\text {fundamental }}(t) & =a_{1} \cos \left(\frac{\omega}{n} t\right)+b_{1} \sin \left(\frac{\omega}{n} t\right) \\
& =\sqrt{a_{1}^{2}+b_{1}^{2}} \cos \left(\frac{\omega}{n} t+\psi\right)
\end{aligned}
$$

with

$$
\psi=\tan ^{-1}\left(\frac{-b_{1}}{a_{1}}\right)+\pi\left(\text { if } a_{1}<0\right)
$$

The instant on which the mass position reaches a maximum is called $t_{1}$. Hence, the phase of the mass position at $t_{1}$ is equal to zero:

$$
\begin{aligned}
\frac{\omega}{n} t_{1}+\psi & =0 \quad \bmod [2 \pi] \\
\Rightarrow t_{1} & =-\psi \frac{n}{\omega} \quad \bmod \left[2 \pi \frac{n}{\omega}\right]
\end{aligned}
$$

The excitation is a monochromatic acceleration $A \cos (\omega t)$. Then, the excitation displacement is $A / \omega^{2} \cos (\omega t+\pi)$. The phase of the excitation displacement when the position of the mass reaches a maximum (at $\left.t_{1}\right)$ is therefore:

$$
\begin{aligned}
\text { Phase } & =\omega t_{1}+\pi \quad \bmod [2 \pi] \\
& =-n \psi+\pi \quad \bmod [2 \pi]
\end{aligned}
$$

Figure 9(b) details the value of this phase for each behavior. If this phase is zero, then the displacement of the excitation reaches a maximum when the position of the mass reaches a maximum. If this phase is $\pi$, then the displacement of the excitation reaches a minimum when the position of the mass reaches a maximum. It is important to note here that the calculated phase is the phase of the excitation displacement. This phase is generally positive: the frame displacement is ahead of the mass displacement.

The powers shown in Figure 9(d) are split in two different types: the mean extracted power and the mean harvested power. The mean extracted power corresponds to the sum of all the extracted energy during one period of the mass oscillation when the switches are closed (four extractions in total). This sum is then averaged over the time of this period:

$$
\left\langle P_{\text {extracted }}\right\rangle=\frac{4}{2} C_{0}\left(v_{\max }-v_{\min }\right)^{2} \frac{2 \pi \omega}{n}
$$

With $v_{\max }$ and $v_{\min }$ the maximal and minimal voltage of the Piezo harvester in open circuit, $\omega$ the excitation angular frequency and $n$ the order of the considered subharmomnic behavior. The mean harvested power corresponds to the mean power converted in the load resistance $R$ :

$$
\left\langle P_{\text {harvested }}\right\rangle=\frac{v_{D C}^{2}}{R}
$$

Contrary to the mean extracted power, the mean harvested power takes into account the electrical losses during the energetic transfers from the piezoelectric converter to the capacitor through the inductance. The experimental analysis evaluates both types of power while the theoretical analysis only predicts the extracted power as it does not consider those electrical losses. The experimental measurements show a mean harvested power of between $50 \%$ and $90 \%$ of the mean extracted power (50\% for the subharmonic 3 behavior at $44 \mathrm{~Hz}$ or for the harmonic 1 behavior at $16 \mathrm{~Hz}$ and $90 \%$ for the subharmonic 3 behavior at $58 \mathrm{~Hz}$ or for the harmonic 1 behavior at $34 \mathrm{~Hz}$ ).

In Figure 9(b), the phase of the excitation displacement when the position of the mass reaches a maximum shows 

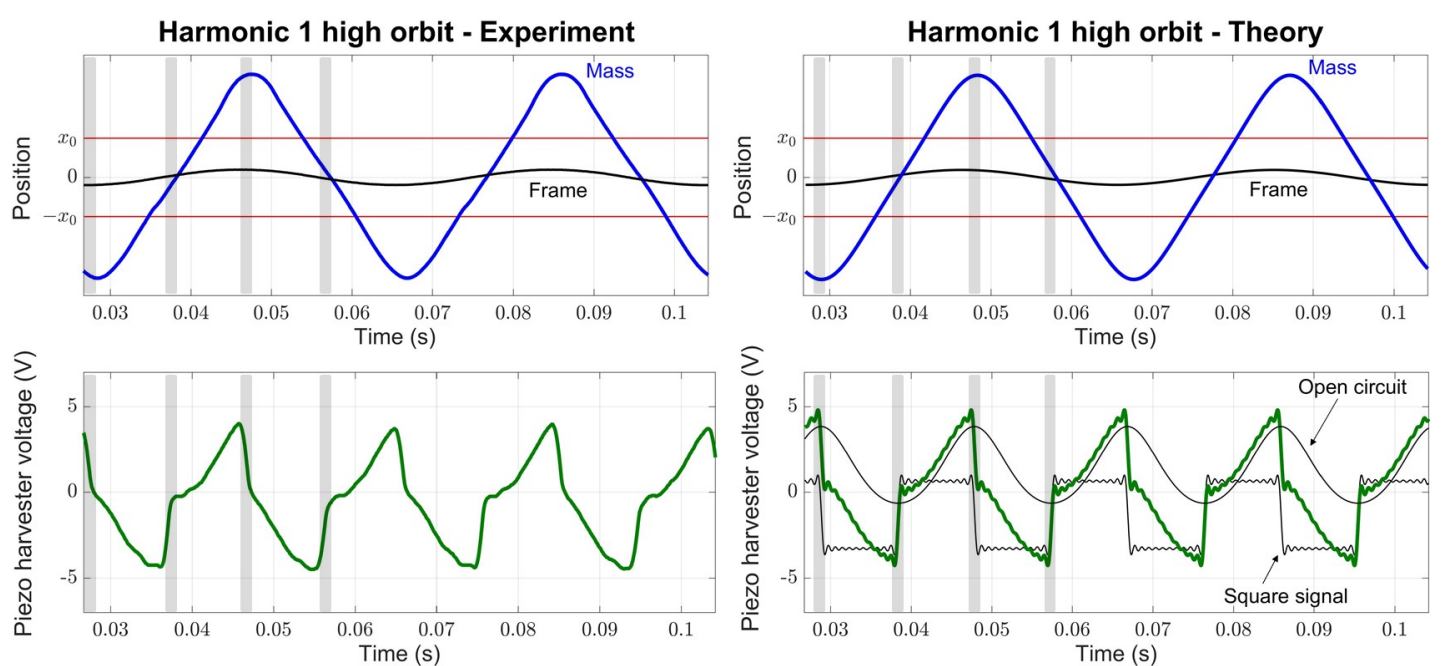

Figure 7: Time signal examples for harmonic 1 high orbit of the bistable harvester coupled to SECE circuit for an excitation of $2.5 \mathrm{~m} . \mathrm{s}^{-2}$ at $26 \mathrm{~Hz}$.
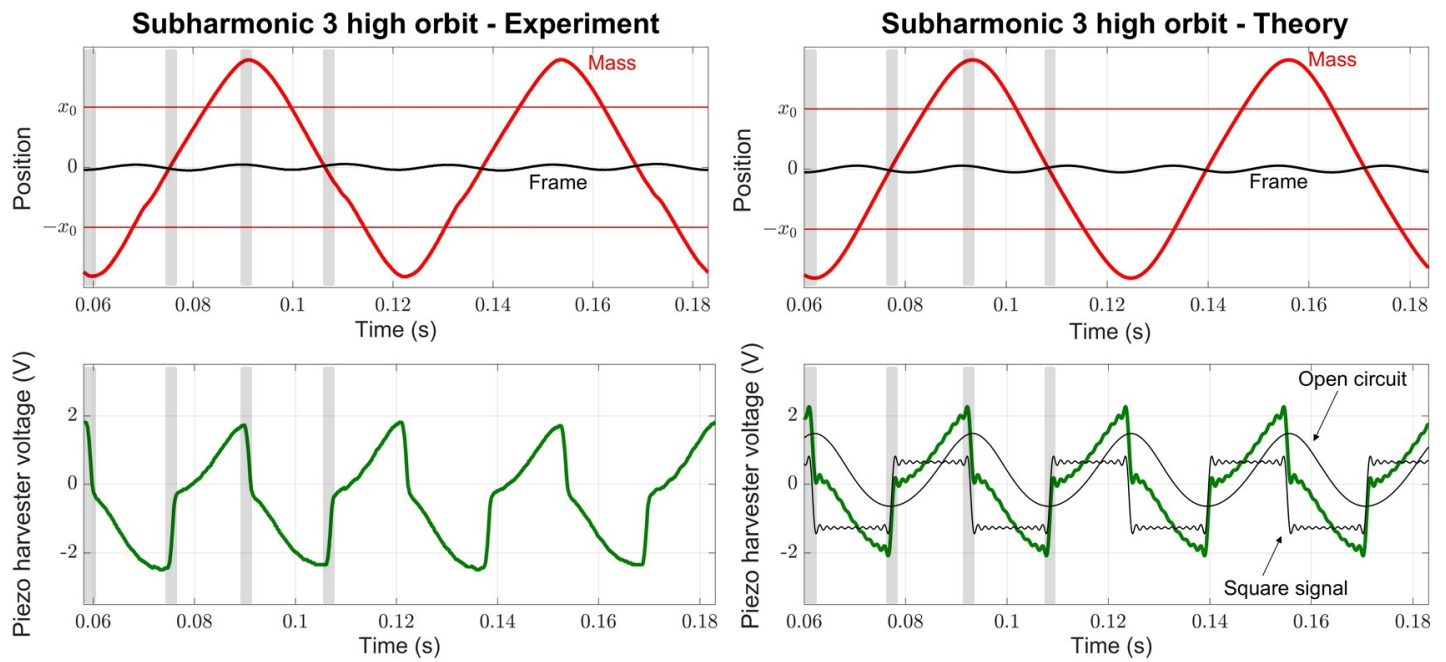

Figure 8: Time signal examples for subharmonic 3 high orbit of the bistable harvester coupled to SECE circuit for an excitation of $2.5 \mathrm{~m} . \mathrm{s}^{-2}$ at $48 \mathrm{~Hz}$.

an interesting particularity: it increases monotonously from zero at the beginning of the theoretical frequency range of each behavior until $\pi / 2$ at the end of their frequency range. This particularity have been highlighted by Harne et al. for harmonic 1 high orbit [19] and extended by the authors to all the odd subharmonic behaviors [16]. When the bistable harvester stabilizes on one of these behaviors, it is now possible to determine if the harvester is close to the beginning or close to the end of its theoretical frequency range measuring the phase of the excitation displacement when the position of the mass reaches a maximum. This phase can therefore be used to characterize the theoretical cutting frequency of these behaviors (i.e., the end of the theoretical frequency range): the latter is reached if and only if the phase of the excitation displacement is equal to $\pi / 2$ when the position of the mass reaches a maximum.

In Figure 9(c), the curves indicate the stability robustness of each behavior so their capacity to handle external disturbances without falling on a low orbit. The threshold above which the behavior will be considered as robust enough for energy harvesting in real conditions is determined ensuring a good correlation between the experimental cutting frequencies and the model predictions. Indeed, the theoretical cutting frequencies (defined by $\pi / 2$ phases) could not have been reached experimentally. The measured frequency ranges of the different behaviors are systematically shorter than the theoretical predictions of the model which does not include the stability robustness. When this criterion is added, the experiment and the theory finally show similar results, thus validating the relevance of the mathematical model developed for the bistable harvester coupled to SECE circuit.

\section{Comparison with and without the SECE circuit}

Figure 10 shows a comparison between the frequency responses of the bistable harvester coupled to the SECE interface circuit (color curves) and the bistable harvester directly connected to the resistive load (light curves). These frequency responses only detail the most interesting behaviors for energy harvesting (harmonic 1 and subharmonic 3 behaviors [15]) and only keep the parts of 

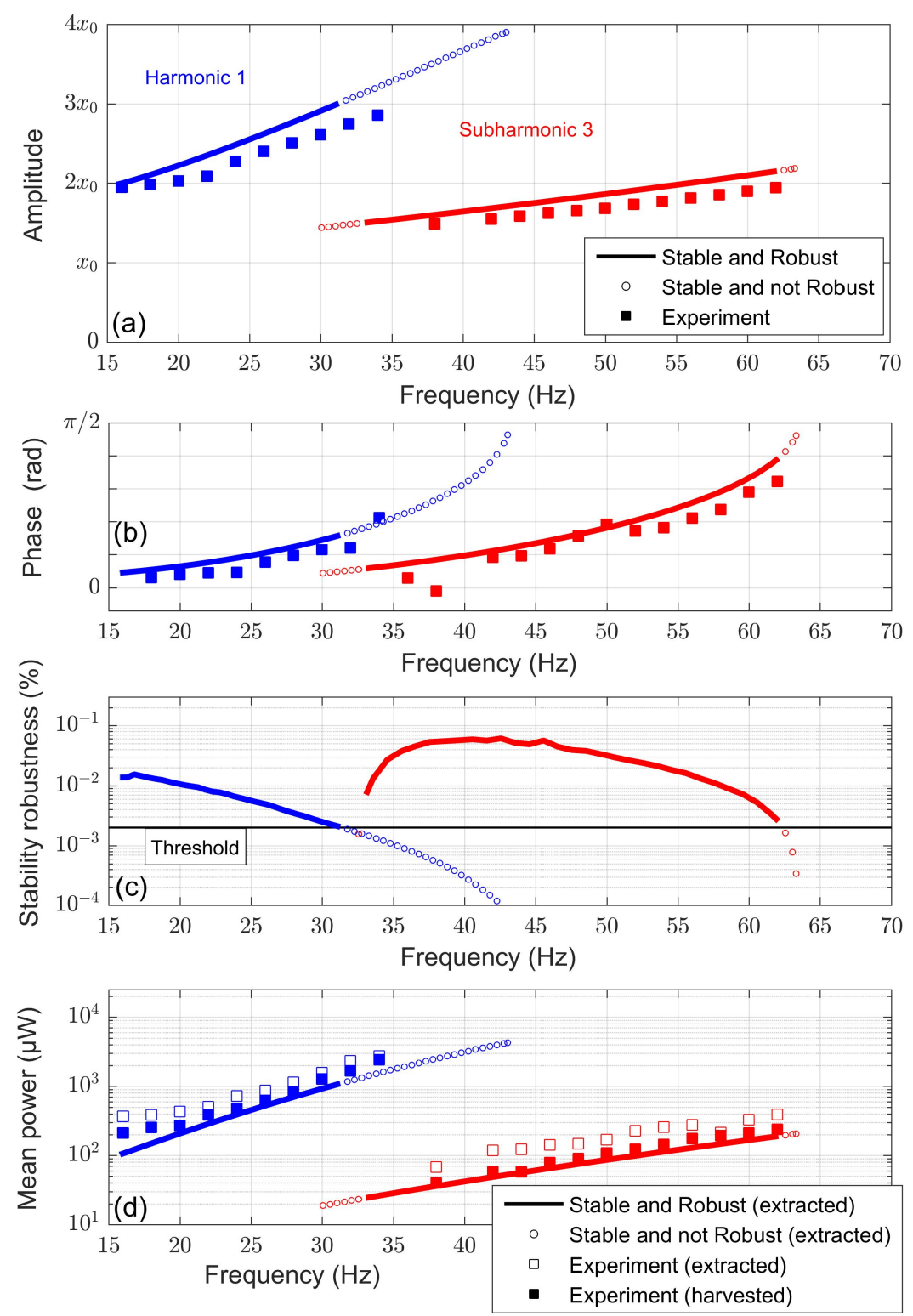

Figure 9: Theoretical and experimental frequency responses of the bistable harvester coupled to SECE circuit for an excitation amplitude of 2.5 m.s $s^{-2}$ : (a) mass oscillations amplitude for the different behaviors (b) phase of the excitation displacement when the position of the mass reaches a maximum (c) stability robustness (d) mean extracted power (theory and experiment) and mean harvested power (experiment).

their frequency range which are defined as stable (with the stability to small disturbances analysis) and robust enough to be maintained over time in real conditions (with the stability robustness analysis). The theoretical model used to predict the frequency response of the bistable harvester directly connected to the resistive load is similar to the one developed here. It corresponds to the model used in a previous study for a bistable harvester with electromagnetic conversion [16], adapted to piezoelectric conversion. The resistance has been chosen to maximize the mean power converted (impedance adaptation: $R=1 / C_{0} \omega_{0}=7.83 \mathrm{k} \Omega$ ).

For a given behavior at a given frequency, the mean extracted power appears to be bigger with the SECE circuit. Reasoning on the theoretical curves, for harmonic 1 behavior, adding the SECE circuit leads to a multiplication of the mean extracted power by 1.4 at $15 \mathrm{~Hz}(103 \mu \mathrm{W}$ compared to $75 \mu \mathrm{W}$ without the SECE circuit) and by
2.2 at $30 \mathrm{~Hz}$ (1024 $\mu \mathrm{W}$ compared to $467 \mu \mathrm{W}$ without the SECE circuit). For subharmonic 3 behavior, adding the SECE circuit leads to a net gain of 1.3 at $33 \mathrm{~Hz}(24 \mu \mathrm{W}$ compared to $19 \mu \mathrm{W}$ without the SECE circuit) and 1.7 at $62 \mathrm{~Hz}(190 \mu \mathrm{W}$ compared to $113 \mu \mathrm{W}$ without the SECE circuit).

The maximum mean extracted power is similar with the two circuits: $1024 \mu \mathrm{W}$ at $30 \mathrm{~Hz}$ (harmonic 1) compared to $1174 \mu \mathrm{W}$ at $40 \mathrm{~Hz}$ (harmonic 1) without the SECE circuit (value taken from the theoretical curves).

On the other hand, the SECE circuit reduces the useful frequency range of each behavior (i.e., the frequency range stable and robust). Reasoning again with the theoretical curves, the frequency range of harmonic 1 behavior is divided by 1.5 (from $25 \mathrm{~Hz}$ to $16 \mathrm{~Hz}$ ) while the frequency range of subharmonic 3 behavior is divided by 1.5 (from $45 \mathrm{~Hz}$ to $29 \mathrm{~Hz}$ ). This reduction in 

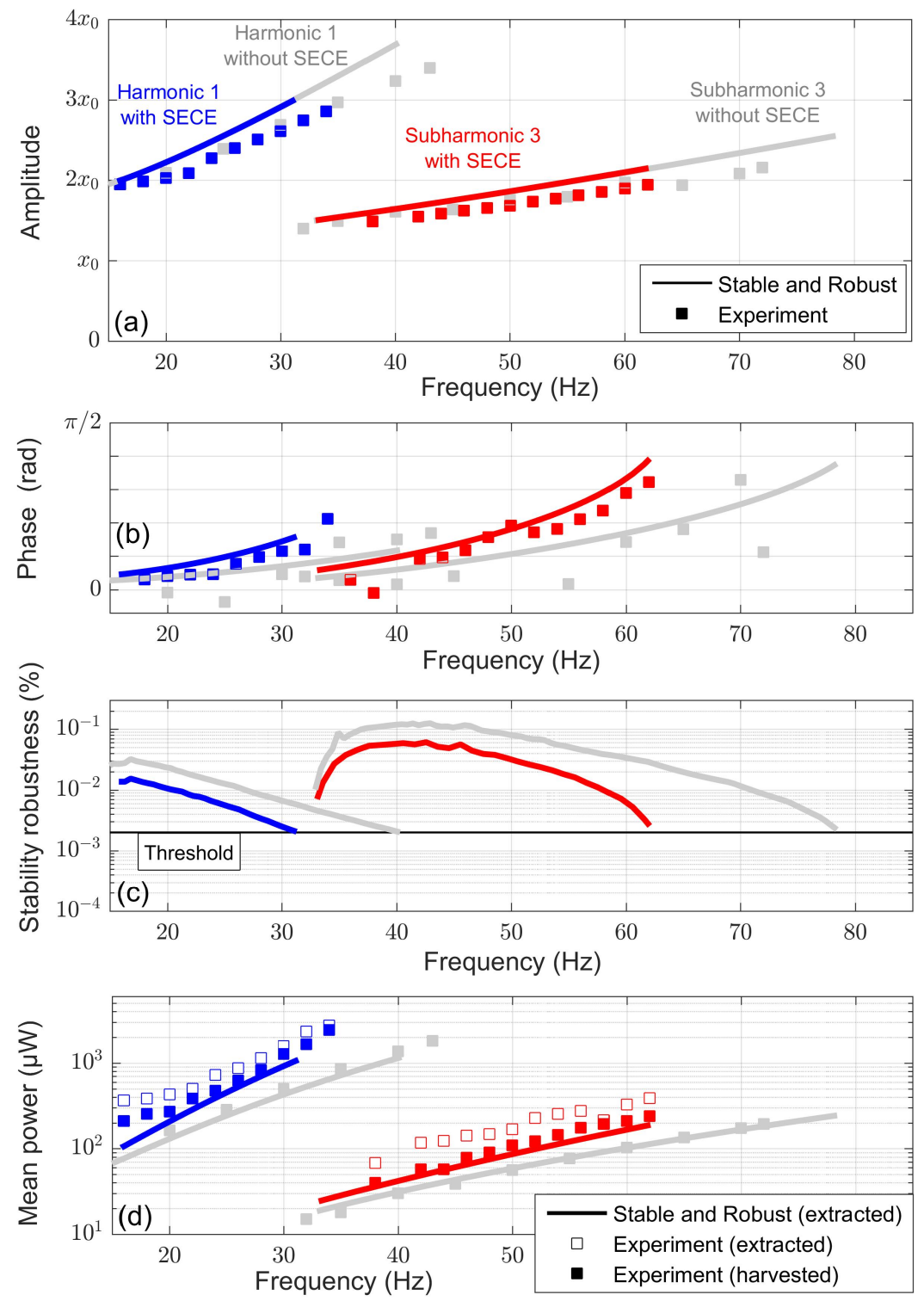

Figure 10: Theoretical and experimental frequency responses of the bistable harvester coupled to SECE circuit (color curves) or directly coupled the resistive load (light curves) for an excitation amplitude of $2.5 \mathrm{~m} \cdot \mathrm{s}^{-2}$ : (a) mass oscillations amplitude for the different behaviors (b) phase of the excitation displacement when the position of the mass reaches a maximum (c) stability robustness (d) mean power.

frequency range is due to the global reduction in the stability robustness of each behavior when adding the SECE circuit (Figure 10(c)). Indeed, the latter increases the mean extracted power without changing the amplitude of the mass oscillation (Figure 10(a)). The extra power therefore comes from the vibrating source: the mechanical coupling between the bistable harvester and the source is increased. Thus, the phase of the excitation displacement when the position of the mass reaches a maximum is getting closer to $\pi / 2$ (Figure 10(b)). The bistable harvester extract more energy from the source but its behaviors get closer to their theoretical cutting frequency, thus reducing their stability robustness and therefore, their accessible frequency range.

The frequency range on which the bistable harvester offers a mean extracted power above $100 \mu \mathrm{W}$ (mean power required to supply a wireless sensor consuming $450 \mathrm{~mJ}$ per transmitted packet [20] with, on average, one packet sent every $1.25 \mathrm{~h}$ ) also decreases when adding the SECE circuit. It goes for theoritical curves from $41 \mathrm{~Hz}$ without the SECE circuit $(22 \mathrm{~Hz}$ for the harmonic 1 behavior and $19 \mathrm{~Hz}$ for the subharmonic 3 behavior) to $26 \mathrm{~Hz}$ with the SECE circuit $(16 \mathrm{~Hz}$ for the harmonic 1 behavior and $10 \mathrm{~Hz}$ for the subharmonic 3 behavior).

The conclusion of this comparison is therefore mixed. The SECE circuit is profitable on the frequency range $[15 \mathrm{~Hz}-31 \mathrm{~Hz}]$ and $[41 \mathrm{~Hz}-62 \mathrm{~Hz}]$ offering mean extracted powers 1.3 to 2.2 times bigger. Connecting the bistable harvester directly to the resistive load is profitable on the frequency range $[32 \mathrm{~Hz}-40 \mathrm{~Hz}]$ and $[63 \mathrm{~Hz}-78 \mathrm{~Hz}]$ where it allows to reach behaviors that are not robust enough with the SECE circuit to be maintained over time. In terms of integration, the SECE circuit is nevertheless advantageous thanks to its continuous output voltage $v_{D C}$ and thanks to the fact that it allows the harvester and the load to be decoupled. Thus, the 
SECE circuit is adapted to supply energy to wireless sensors without worrying about any particular impedance adaptation to maximize the extracted power from the vibration source.

\section{Summary and Conclusion}

The objective of this study was to construct an analytical model able to predict the frequency response of a bistable harvester coupled to a SECE interface circuit. The first part thus details the construction of the model which includes the study of the subharmonic behaviors that widen the global bandwidth of the harvester (in particular the subharmonic 3 behavior). The different steady-state high orbits (inter-well behaviors) are then characterized by their stability to small disturbances and by their stability robustness reflecting their capability to handle external disturbances in real conditions without falling on a low orbit. The frequency response predicted by this mathematical model showed a good agreement with the experimental analysis conducted in this study, thus validating its relevance.

Then, the analytical model has been used to compare the bistable harvester coupled to the SECE circuit and the bistable harvester directly connected to the load. The results show that the mean extracted power is 1.3 to 2.2 times bigger with the SECE circuit all over its reachable frequency range (stable and robust enough). However, this reachable frequency range is divided by $1.5 \mathrm{com}-$ pared to the one reachable with a direct connection to the load. The use of SECE circuit therefore increases the mean extracted power but decreases the global bandwidth of the bistable harvester. The SECE circuit remains nevertheless advantageous in terms of integration thanks to its continuous output voltage, ideal for wireless sensors supply, and thanks to the decoupling that it offers between the harvester and the load (no impedance matching required to maximize the extracted power).

It would be now interesting, in a future work, to extend the analytical model developed here to other AC-DC circuits such as the bridge rectifier or the SSHI circuit in order to establish a fair comparison between them for bistable harvester. It would also be interesting to investigate the influence on the bistable harvester of a partial SECE circuit which would only extract a part of the entire energy of the piezoelectric converter at each voltage extrema. The mean power extracted could therefore be decreased in order to adjust the ratio between extracted power and bandwidth of the harvester.

\section{Acknowledgment}

The authors acknowledge the support of Région Auvergne-Rhône-Alpes through the ARC 4 Energies program.

\section{Compliance with Ethical Standards}

Conflict of interest. The authors declare that they have no conflict of interest.

\section{References}

[1] J. Siang, M.H. Lim, and M. Salman Leong. Review of vibration-based energy harvesting technology : Mechanism and architectural approach. International Journal of Energy Research, pages 1-28, 2018.

[2] M.F. Daqaq, R. Masana, A. Erturk, and D.D. Quinn. On the role of nonlinearities in vibratory energy harvesting: A critical review and discussion. Applied Mechanics Reviews, 66:40801, 2014.

[3] R.L. Harne and K.W. Wang. A review of the recent research on vibration energy harvesting via bistable systems. Smart Materials and Structures, 22:23001, 2013.

[4] D. Guyomar, A. Badel, E. Lefeuvre, and C. Richard. Toward Energy Harvesting Using Active Materials and Conversion Improvement by Nonlinear Processing. In IEEE transactions on ultrasonics, ferroelectrics, and frequency control, volume 52, pages 584595, April 20052005.

[5] G.W. Taylor, J.R. Burns, S.M. Kammann, W.B. Powers, and T.R. Welsh. The Energy Harvesting Eel : A Small Subsurface Ocean / River Power Generator. In IEEE Journal of Oceanic Engineering, volume 26, pages 539-547, October 2001.

[6] E. Lefeuvre, A. Badel, C. Richard, L. Petit, and D. Guyomar. A comparison between several vibration-powered piezoelectric generators for standalone systems. Sensors and Actuators A: Physical, 126:405-416, 2006.

[7] E. Lefeuvre, A. Badel, C. Richard, and D. Guyomar. Piezoelectric energy harvesting device optimization by synchronous charge extraction. Journal of Intelligent Material Systems and Structures, 16:865-876, 2005.

[8] C. Richard, D. Guyomar, D. Audigier, and H. Bassaler. Enhanced semi passive damping using continuous switching of a piezoelectric device on an inductor. In Proceedings of SPIE : Smart Structures and Materials, volume 3989, pages 288-299, 27 April 2000.

[9] D. Guyomar and M. Lallart. Recent Progress in Piezoelectric Conversion and Energy Harvesting Using Nonlinear Electronic Interfaces and Issues in Small Scale Implementation. Micromachines, 2:274294, 2011. 
[10] L Wu, X. Do, S. Lee, and D.S. Ha. A self-powered and optimal sshi circuit integrated with an active rectifier for piezoelectric energy harvesting. IEEE Transactions on Circuits and Systems I: Regular Papers, 64:537-549, 2017.

[11] W.L. Shareef, A.and Goh, S. Narasimalu, and Y. Gao. A rectifier-less ac-dc interface circuit for ambient energy harvesting from low-voltage piezoelectric transducer array. IEEE Transactions on Power Electronics, 34:1446-1457, 2019.

[12] W.Q. Liu, A. Badel, F. Formosa, Y.P. Wu, and A. Agbossou. Wideband energy harvesting using a combination of an optimized synchronous electric charge extraction circuit and a bistable harvester. Smart Materials and Structures, 22:125038, 2013.

[13] Y.Y. Chen, D. Vasic, and Y.P. Liu. Study of a piezoelectric switching circuit for energy harvesting with bistable broadband technique by work-cycle analysis. Journal of Intelligent Material Systems and Structures, 24:180-193, 2013.

[14] L. Alari, J.P. Udani, A.F. Arrieta, and F. Braghin. Synchronized switch technique based on dynamical state of bi-stable energy harvesters. In Proceedings of SPIE, volume 10595, pages 1-13, 5 March 2018.

[15] T. Huguet, A. Badel, and M. Lallart. Exploiting bistable oscillator subharmonics for magnified broadband vibration energy harvesting. Applied Physics Letters, 111:173905, 2017.

[16] T. Huguet, A. Badel, O. Druet, and M. Lallart. Drastic bandwidth enhancement of bistable energy harvesters: Study of subharmonic behaviors and their stability robustness. Applied Energy, 226:607-617, 2018.

[17] W.Q. Liu, A. Badel, F. Formosa, Y.P. Wu, and A. Agbossou. Novel piezoelectric bistable oscillator architecture for wideband vibration energy harvesting. Smart Materials and Structures, 22:035013, 2013.

[18] A. Badel and E. Lefeuvre. Nonlinear Conditioning Circuits for Piezoelectric Energy Harvesters. In E. Blokhina, A. El Aroudi, E. Alarcon, and D. Galayko, editors, Nonlinearity in Energy Harvesting Systems Nonlinear, pages 321-359. Springer, Cham, 2016.

[19] R.L. Harne, M. Thota, and K.W. Wang. Concise and high-fidelity predictive criteria for maximizing performance and robustness of bistable energy harvesters. Applied Physics Letters, 102:053903, 2013.

[20] A. Gutiérrez, C. Gonzàlez, J. Jiménez-Leube, S. Zazo, N. Dopico, and I. Raos. A Heterogeneous Wireless Identification Network for the Localization of Animals Based on Stochastic Movements. Sensors, 9:3942-3957, 2009. 